\title{
Inverse identification of process variations for thin steel sheet bending
}

\author{
Jos Havinga a , Ton van den Boogaard \\ University of Twente, Nonlinear Solid Mechanics \\ PO Box 217, 7500AE, Enschede, The Netherlands
}

ag.t.havinga@utwente.nl

\begin{abstract}
Keywords: Inverse analysis, sheet bending, proper orthogonal decomposition, process variation, tooling alignment
\end{abstract}

\begin{abstract}
The stability of a metal forming production process is influenced by several sources of scatter such as variation of material and lubrication properties. Identification of the sources of variation is needed to optimize the process settings or to design a control strategy for the process. Many engineers point out sources of variation by experience, but in complex cases a computational identification algorithm may be used to investigate the process.

When using parameter estimation in a control system, process forces can be used for the estimation. However, many parameters may influence the process forces. Therefore extensive models are needed to be able to identify the process parameters, including parameters such as tooling misalignment.

In the current work, a thin steel flap bending process is studied. Measurements from an industrial press are used to identify the process parameters. A metamodel based inverse analysis procedure is used. The procedure is extended with proper orthogonal decomposition (POD) of the force curves to increase its convergence rate.
\end{abstract}

\section{Introduction}

One of the major challenges within the field of metal forming research is to understand and to decrease the output variation of forming processes. Variation of process inputs such as material properties, temperature or friction properties cause variation of the final product geometry or even production failure. One approach adopted in research and industry to increase the process robustness is to quantify the input variation and take it into account during the process design. A robust design is found when the requirements of the product are met even under the influence of the input variation.

In some cases the production requirements are so tight that further improvements of the production process are needed. Applying real time control to the production process may help to reach the desired production accuracy. To do so, process monitoring is needed through in-line process measurements. The quality requirements may be directly measured with an in-line quality control system that measures the final geometry of every product. On the other hand, secondary process data such as process forces or tooling temperature may be measured and used in the control system.

However, it is not a straightforward task to relate secondary process measurements to the final quality of the product. A possible approach is to produce a large number of products and to use statistical methods to find relations between the secondary measurements and the product quality. Such approaches have been successfully applied in the semiconductor industry [1]. The drawback is the large number of products needed to be able to retrieve these relations. Another approach is to construct a simulation model of the process and to use inverse analysis methods to get an estimate of the process variations. Several successful studies of inverse analysis in metal forming are known. However, inverse analysis is mostly used for material model fitting [2, 3, 4]. Well-controlled experiments are used to fit material parameters through inverse analysis. These are usually well-posed optimization problems. However, in an industrial production process the number of variation parameters may be large and it may be difficult to distinguish the effects of different parameters. 
In this study an attempt is made to use inverse analysis methods with data retrieved from an industrial bending process. An extensive model of the process is built, including many unknown parameters such as material parameters, tooling elasticity and tooling misalignment. Measured force curves are used to identify these parameters. Two different inverse analysis approaches are tested. Both approaches use radial basis function (RBF) metamodels of the process. In one case the value of the error-function is directly interpolated, in the second case POD of the force curve is used to predict the full force curve at untried points.

In the following section the finite element (FE) model of the bending process is presented. Then results of the screening study are shown. Thereafter the inverse analysis algorithms are explained, followed by the results and conclusion.

\section{Bending model}

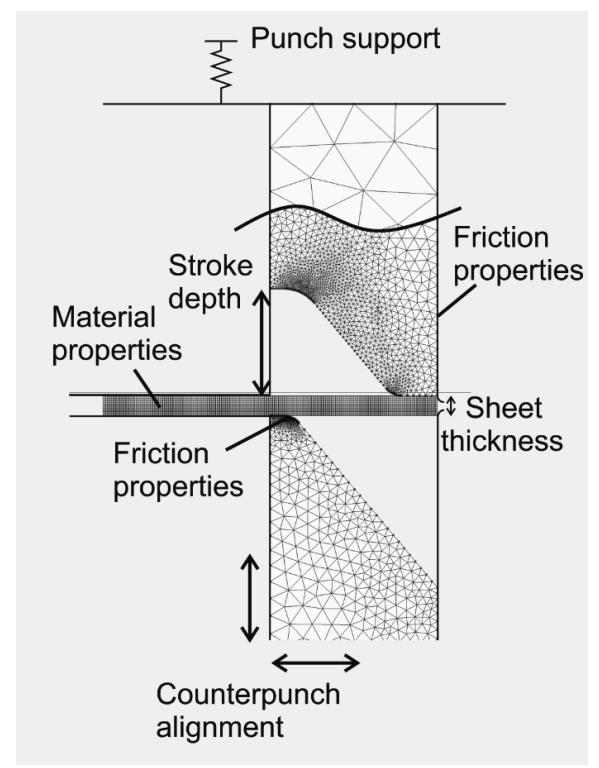

Fig. 1: Model parameters

The studied bending process consists of an over-bending and a back-bending stage of a $0.3 \mathrm{~mm}$ thick and $1.5 \mathrm{~mm}$ wide AISI 420 steel flap, with a production rate of 60 products per minute. During over-bending the bending force is measured. The depth of the stroke during back-bending can be controlled from product to product, enabling process control. In this work only the first bending stage is studied. Understanding of the effect of different parameters on the bending force is needed to be able to interpret the bending forces and use them in the control of the back-bending stage.

Creating a model of a process by definition means simplification. The physical connection of the process with its environment is interpreted through boundary conditions. Behavior of material and lubrication is gathered in mathematical models. The tooling geometry is interpreted from technical drawings and transferred to the simulation model. While simplification is needed to make a workable model, the risk of oversimplification arises. Especially when using measurements from an industrial setting, certain influencing parameters may be overlooked, making it impossible to find the correct input parameters. Therefore parameters such as play, tooling misalignment and tooling compliance must be taken into account. By performing a screening study, the most influential model parameters can be selected for the inverse analysis.

A 2D model of the first bending step has been built with the MSC.Marc FE software (Fig. 1). The punch and counterpunch are modeled with solid elastic elements. The support of the punch has been modeled as a spring with two regions, each one with a different stiffness, to reflect potential play and machine compliance (Fig. 2). Vertical and horizontal alignment of the die and the depth of the punch stroke are taken into account as possible misalignment parameters.

The vertical movement of the press is controlled by a crank system. Therefore the press slows down while approaching its deepest point. Due to the strainrate sensitivity of the used mate-

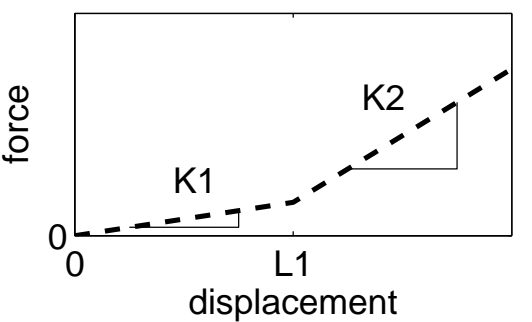

Fig. 2: Parameters of spring at punch support rial, the maximum force is reached before reaching the deepest point of the stroke. To include this effect on the shape of the bending force curve in the model, the strainrate sensitivity of the material has been modeled with a power law (Eq. 1). The parameters of the model have been included in the inverse analysis study. A tabular hardening model for zero strainrate $\sigma_{y}(\epsilon \mid \dot{\epsilon}=0)$ is used. 


$$
\sigma_{y}(\epsilon, \dot{\epsilon})=\sigma_{y}(\epsilon \mid \dot{\epsilon}=0) \cdot\left(1+\left(\frac{\dot{\epsilon}}{\dot{\epsilon}_{0}}\right)^{z}\right)
$$

The number of elements in the simulation is 3600 for the sheet and 5376 for the tooling. The wall time of a simulation is around 6 to 8 minutes on a standard workstation.

\section{Influence of parameters}

A screening study has been performed to select the parameters that influence the bending force curve. In Fig. 3, the influence of the selected parameters on the bending force is shown. It can be seen that all the selected parameters have a significant influence on the force curve. Some parameters have a comparable effect on the force curve, such as sheet thickness and friction between the sheet and the punch. Furthermore it can be noticed that misalignment parameters such as the depth of the punch stroke and the horizontal and vertical counter-punch alignment have a strong effect on the force curve. Omitting these parameters in the inverse analysis would likely lead to a wrong solution. Therefore all parameters with a significant influence on the force curve have been selected for the inverse analysis of the bending force.
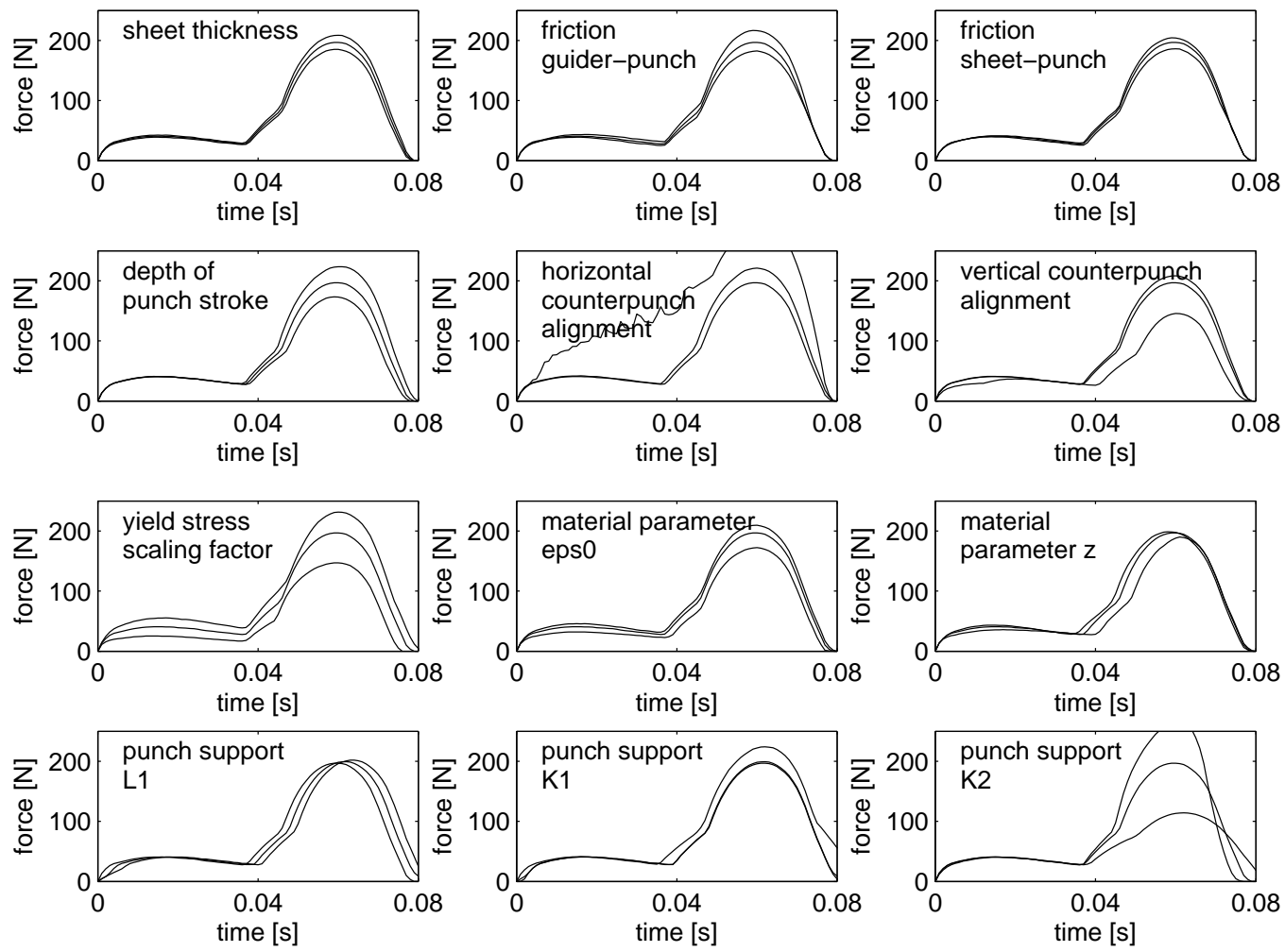

Fig. 3: Influence of selected parameters on the bending force. All parameters have been set to the minimum, the maximum and the nominal values.

\section{Inverse analysis}

The 12 parameters selected in the screening study span a huge parameter space that has to be investigated in the inverse analysis study. To do so, a metamodel based optimization scheme is used. The 
objective of the inverse analysis is to minimize the error between the experimental force curve and the force curve obtained from the simulation. The following error-function is used:

$$
e=\sum_{t=0}^{100}\left|f_{\exp }(0.001 t)-f_{\text {sim }}(0.001 t)\right|
$$

To start with, an initial design of experiments (DOE) is built. A two-level resolution IV $2^{12-6}$ fractional factorial design has been combined with a latin hypercube design of 100 points. Due to some simulation crashes the initial DOE size is 148 . The following step is to fit an interpolating metamodel through the DOE points. The chosen metamodel type is a radial basis function (RBF) with multiquadric basis functions. The leave-one-out-cross-validation value can be obtained without extra computational cost. It is used to compute an estimate of the prediction error of the metamodel at the untried DOE points. Now a set of parameters for a new simulation can be selected. The point with the maximum expected improvement is selected, as proposed in [5]. This cycle is repeated a predefined number of times, aiming to identify the parameters of the experiment. The algorithm is summarized in Fig. 4a.

To study the convergence rate of the inverse analysis algorithm, an improvement to the approach has been made. Instead of interpolating the values of the error-function at the DOE points, a predictive model of the full force curve is built. The predictive model is based on proper orthogonal decomposition (POD) of the force curves at the DOE points. With the POD approach, the force curves can be rewritten as a sum of basis vectors times a set of weighting coefficients. The weigthing coefficients are interpolated with RBF metamodels, resulting in a predictive model for the full force curve. For a more detailed explanation of the method, see [6]. The predictive model of the force curve can be used to predict the value of the error-function for any untried set of parameters. Therefore it can be used to replace the predictive model of the error-function in the maximization of the expected improvement. The extended algorithm is summarized in Fig. $4 \mathrm{~b}$.

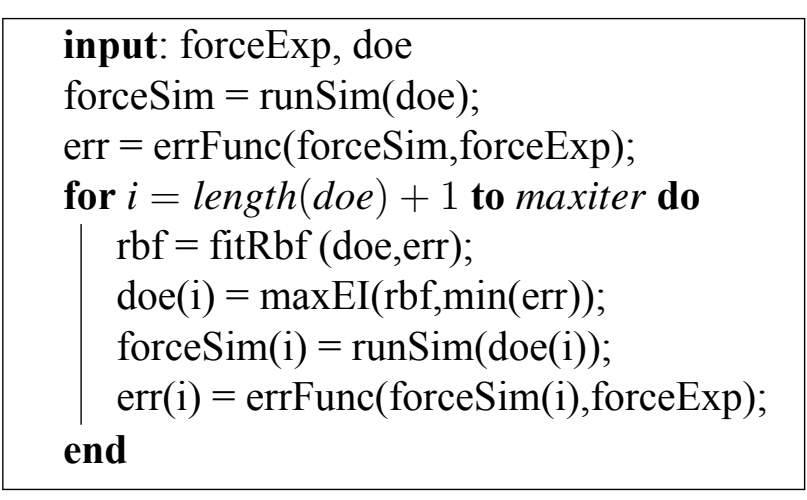

(a) Standard inverse analysis

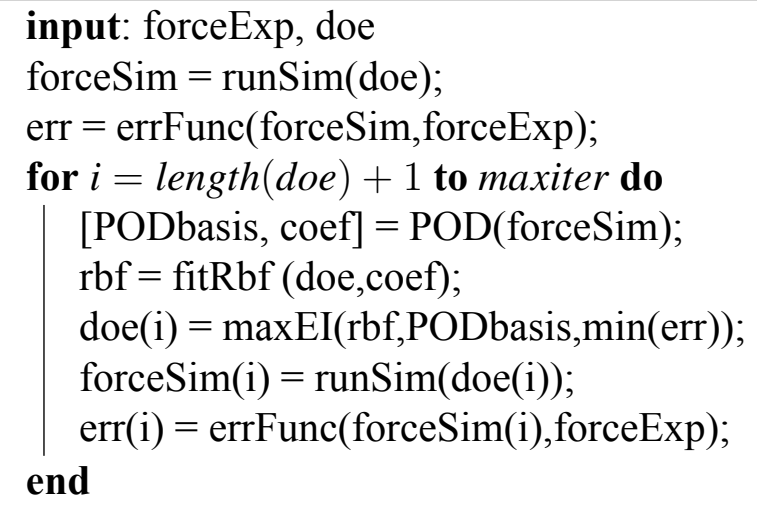

(b) POD inverse analysis

Fig. 4: A summary of the algorithms used for inverse analysis.

\section{Results}

Three distinct force curves have been selected from the experimental dataset. Both inverse analysis methods have been used to identify the parameter set of these curves. After 125 sequential improvement iterations the best results of the different approaches are compared. The force curves with the lowest error can be seen in Fig. 5. During the first 0.04 seconds, free bending occurs. After 0.04 seconds, the tip of the flap touches the counter-punch, causing a strong increase of the force. The maximum force is reached just before the deepest point of the punch. For force curve 3, an increase 
of the force during free bending is caused by misalignment of the counter-punch. However, this was not identified with the standard approach.

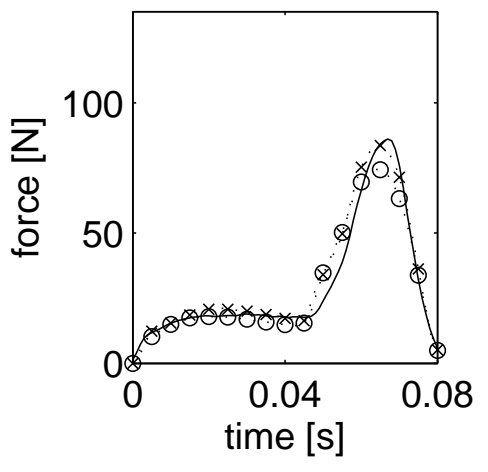

(a) Force curve 1

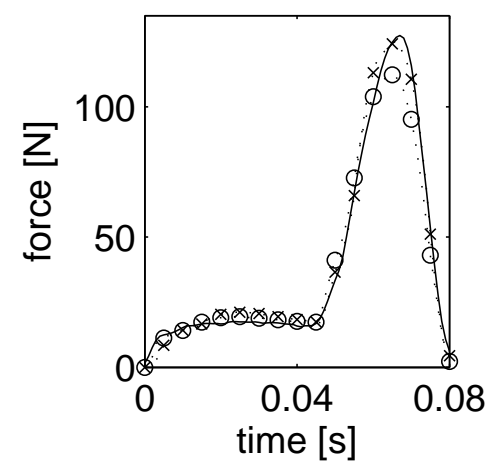

(b) Force curve 2

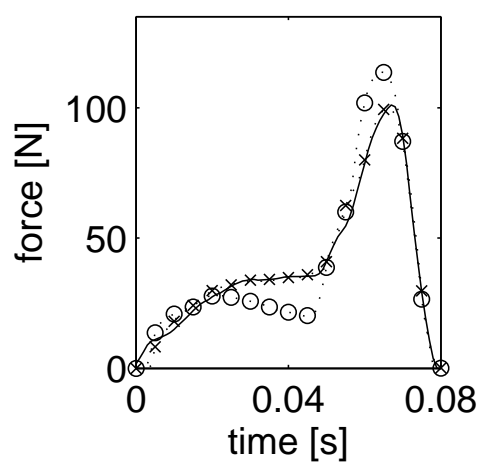

(c) Force curve 3

Fig. 5: Bending forces: testdata (solid line), best fit with standard approach (dotted line with $\circ$ marker) and best fit with POD approach (dotted line with $\times$ marker).

Concerning the convergence rate of both algorithms, it should be noticed that the results for both approaches have been found with a low number of simulations: only 148 initial simulations plus 125 extra simulations in a 12-dimensional space. However, the amount of improvement during the sequential optimization phase is very different from run to run (Fig 6). The run for force curve 3 with the POD approach shows strong continuous improvement, whereas both approaches do not manage to give much improvement to the fit of force curve 1. For all runs, the POD approach converges faster than the standard approach.

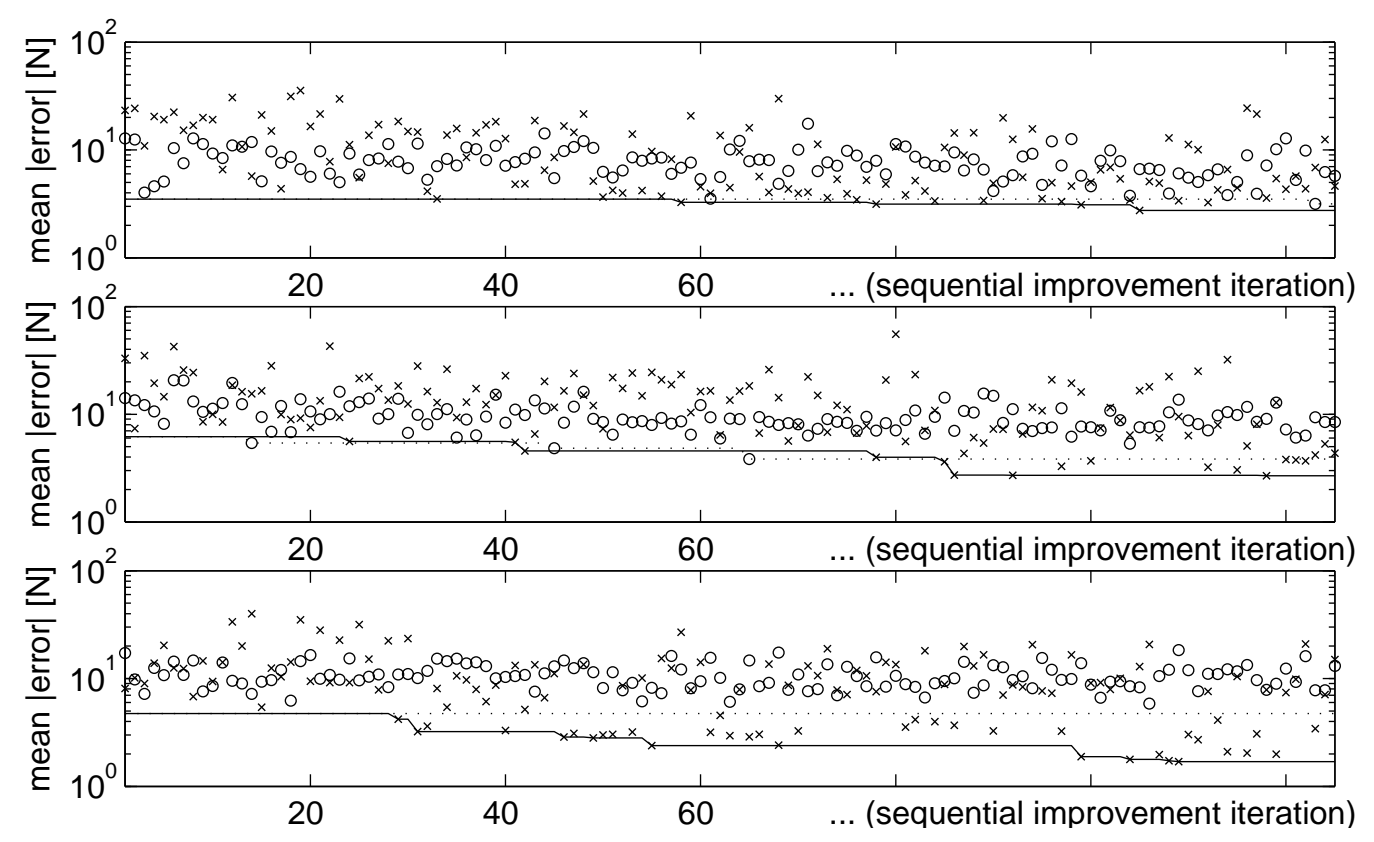

Fig. 6: Error value of all simulations. From top to bottom the convergence for force curves 1 to 3 is shown. The circles show the errors of the standard algorithm, the crosses show the errors of the POD algorithm. The lines give the value of the best solution up to the current sequential improvement iteration: the dotted line for the standard approach and the solid line for the POD approach.

The values of the best set of parameters are shown in Table 1. The standard and the POD approach give very distinct values for most parameters. Therefore it should be concluded that no clear set of parameters has been found. Due to the large number of parameters several solutions of the inverse 
analysis problem can be found with a similar error value. However it can be seen that the POD approach managed to identify the misalignment of the counter-punch for force curve 3 , which causes the higher force in the free bending part.

Table 1: Identified parameters for all force curves with standard and POD approach.

\begin{tabular}{|c|c|c|c|c|c|c|}
\hline & \multicolumn{2}{|c|}{ force curve 1} & \multicolumn{2}{|c|}{ force curve 2} & \multicolumn{2}{|c|}{ force curve 3} \\
\hline & standard & POD & standard & POD & standard & POD \\
\hline sheet thickness [mm] & 0.305 & 0.303 & 0.305 & 0.299 & 0.305 & 0.298 \\
\hline friction guider-punch [-] & 0.264 & 0.150 & 0.294 & 0.231 & 0.010 & 0.064 \\
\hline friction sheet-punch [-] & 0.010 & 0.057 & 0.010 & 0.116 & 0.102 & 0.089 \\
\hline final punch distance $[\mathrm{mm}]$ & 0.317 & 0.305 & 0.280 & 0.287 & 0.330 & 0.314 \\
\hline horizontal couterpunch alignment $[\mu \mathrm{m}]$ & -8.99 & -5.56 & -4.88 & -4.91 & -4.26 & 5.81 \\
\hline vertical counter-punch alignment $[\mu \mathrm{m}]$ & -11.4 & -30.0 & -29.8 & -29.1 & -29.8 & -30.0 \\
\hline yield stress scaling factor [-] & 0.87 & 0.63 & 1.08 & 0.62 & 1.40 & 0.76 \\
\hline $\log 10\left(\right.$ material parameter $\left.\dot{\epsilon}_{0}\right)[-]$ & 2.81 & 3.37 & 5.00 & 4.98 & 1.00 & 4.71 \\
\hline material parameter $z[-]$ & 0.41 & 0.10 & 0.40 & 0.10 & 1.00 & 0.10 \\
\hline punch support L1 [mm] & 0.08 & 0.13 & 0.08 & 0.20 & 0.17 & 0.20 \\
\hline punch support $\mathrm{K} 1$ [N/mm] & 60.3 & 43.6 & 0.0 & 20.6 & 0.0 & 0.6 \\
\hline punch support $\mathrm{K} 2[\mathrm{~N} / \mathrm{mm}]$ & 1000 & 1521 & 2073 & 3514 & 7421 & 3219 \\
\hline
\end{tabular}

\section{Conclusion}

Inverse analysis of an industrial bending process has been performed. Many parameters can be found which influence the force curve. Omitting these parameters makes it impossible to identify the process parameters. Therefore the 12 most important parameters have been included in the inverse analysis study. No clear solution of the problem has been found due to the huge parameter space and interactions between parameters. However it was shown that inclusion of the misalignment parameters is essential for the inverse analysis procedure. Furthermore it was shown that extension of the inverse analysis algorithm with POD of the force curve leads to faster convergence of the inverse analysis algorithm.

\section{Acknowledgement}

The work leading to these results has received funding from the European Community's Seventh Framework Programme under grant agreement n FP7-285030.

MEGaFiT

\section{References}

[1] A.A. Khan, J.R. Moyne and D.M. Tilbury: IEEE Transactions on semiconductor Manufacturing Vol. 20 No. (2007), p. 364-375

[2] P.-A. Eggertsen and K. Mattiasson: Eng. Comput. Vol. 26 No. 2 (2010), p. 159-170

[3] B.E. Kristensen and K.B. Nielsen: Proceedings of the 5th International Conference and Workshop on Numerical Simulation of 3D Sheet Forming Processes (2002), p. 43-48

[4] K.M. Zhao and J.K. Lee: Commun. Numer. Meth. En. Vol. 20 No. 2 (2004), p. 105-118

[5] D. Jones, M. Schonlau and W. Welch: J. Global Optim. Vol. 13 No. 4 (1998), p. 455-492

[6] V. Buljak: FME Transactions Vol. 38 No. 3 (2010), p. 129-136 